\title{
Study of the Interlamellar Folded Structure of Polyethylene as Revealed by Melting Point and Crystallinity
}

\author{
F. J. Baltá Calleja and D. R. Rueda \\ Instituto de Quimica Física "Rocasolano"-C.S.I.C. \\ Serrano 119, Madrid 6, Spain.
}

(Received July 31, 1973)

\begin{abstract}
A modification of Knox's ${ }^{1}$ approach for the estimation of the average number of noncrystalline methylene groups per fold in polyethylene is suggested. The plot of melting point, $T(l)$, from Thomson's equation against inverse of crystallinity, $\alpha$, from a simple model which assumes crystallinity deficiency located at the crystal surface yields a family of straight lines that can be represented by $T(l)=T_{\infty}-K(1-\alpha) / f \alpha . \quad T_{\infty}$ is here the melting point of an infinitely thick crystal, $K$ is a constant, and $f$ is the average number of noncrystalline backbone units per fold contributing to the amorphous layer. Comparison of experimental data with this model shows that $f$ depends upon crystallization conditions annealing treatment, and chain defect content (branching). The obtained results clearly indicate that $f$ is notably smaller for single crystals than for melt-crystallized material, in agreement with previous results from Kawai. ${ }^{20}$ The discussion of $f$ data for single crystals supports the view of a regularly folded surface layer with variable amounts of disorder. The analysis of data derived from the results of Fischer and Schmidt22 in the light of this model indicates that the annealing of crystals provokes an increase in $f$.

KEY WORDS Melting Point / Density / Crystallinity / Branching

Concentration / Crystal Thickness / Methylene Units per Fold /

Single Crystals / Melt-Crystallized Material / Polyethylene /
\end{abstract}

In an earlier publication, Knox ${ }^{1}$ has indicated the possibility of an empirical method which furnishes an estimate of the number of noncrystalline methylene groups per fold in polyethylene (PE). This method, regardless of the structural model adopted, essentially consists of a plot of melting point $T(n)$ derived from the extrapolation of the homologous $n$-alkane series against crystallinity, according to the model:

$$
\alpha=\nu /(\nu+f)
$$

$\nu$ is here the number of lattice backbone units within the crystalline lamellae, $f$ is a parameter equal to the number of nonlattice backbone units contributing to the fold (or simply to the noncrystalline molecule), and $\nu+f=n$. By comparison of experimental crystallinity values of PE for a wide range of varying branching concentrations with data derived from the model it is concluded that around $f=40$ a good fit for all samples is reached. Such a value is roughly in agreement with results suggested by other authors., ${ }^{2,3}$ One ought to expect, however, a certain dependence of branching concentration not only on the crystalline, $l$, but also on the amorphous, $a$, layer-thickness ${ }^{4}$ and consequently in the $f$ value. The collected data by Knox show indeed a decrease of $l$ (decrease in $T(l)$ ) with increasing branching concentration, though they fail to exhibit any variation in $f$. The constant result of $f=40$ is, hence, unreasonably low for small crystallinities and too high for large crystallinities. This situation demands a modification of the above-mentioned approach.

In the present paper a modification of the above model for PE which introduces a justifiable dependence of $f$ on crystallinity is proposed. In addition, new crystallinity data for a series of melt-crystallized PE samples are discussed in the light of the modified model. The analysis of results shows, in fact, that $f$ depends upon crystallization conditions, branching concentration, and molecular weight. 


\section{COMMENT ON THE MODEL}

For simplicity the present approach will not take into account the possible contribution of defect areas within the lattice to the lowering of crystallinity. ${ }^{5,6}$ Furthermore, according to current views $^{6,7}$ it will be assumed that crystallinity deficiency is principally located at the crystal surface, i.e., the two phase concept will be utilized.

The main objection that can be made to the above-mentioned model $^{1}$ is the substitution of $n=\nu+f$ in the expression for the melting point of the $n$-alkane series:

$$
T(n)=139.5-(2500 / n)+\left(8500 / n^{2}\right)
$$

High $f$ values would obviously yield excessively low melting points since $n \gg \nu$. Such an approximation can be only admissible for low $f$ values, i.e., when $n$ approaches $\nu$. In addition, the application of eq 2 to PE is somewhat questionable since this relationship only applies for crystals with extended chains and does not take into account the chain folding at the crystal surface. ${ }^{8}$ A more realistic approach can simply be achieved by a combination of Thomson's equation:

$$
T(l)=T_{\infty}\left(1-2 \sigma / \Delta h_{\mathrm{f}} \rho_{\mathrm{e}} l\right)
$$

and eq 1 with the additional assumption: $l=$ $\nu \times 1.27 \times 10^{-8} \mathrm{~cm} . T(l)$ is here the melting temperature of a crystal with a thickness $l$, given in ${ }^{\circ} \mathrm{K}, T_{\infty}$ and $\Delta h_{\mathrm{f}}$ are the melting temperature $\left({ }^{\circ} \mathrm{K}\right)$ and heat of fusion of an infinitely thick crystal respectively, $\sigma$ is the free energy of the chain folded surface, and $\rho_{\mathrm{c}}$ is the crystal density.

Thus, the substitution of $\nu=\alpha f /(1-\alpha)$ instead of $n$ from eq 1 into eq 3 yields the following hyperbolic dependence of $T(l)$ on $\alpha$ :

$$
T(l)=T_{\infty}-\frac{K(1-\alpha)}{f}\left(\frac{1}{\alpha}\right)=T_{\infty}+\frac{K}{f}-\frac{K}{f}\left(\frac{1}{\alpha}\right)
$$

in which $K=2 \sigma T_{\infty} / \Delta h_{\mathrm{f}} \rho_{\mathrm{c}}\left({ }^{\circ} \mathrm{K} \mathrm{cm}\right)$ and $f$ must be given in $\mathrm{cm}$, i.e., $f$ must be multiplied by a factor $1.27 \times 10^{-8}$. Equation 4 demands, in addition, that for $\alpha=1, T(l)=T_{\infty}$.

The plot of $T(l)$ vs. $1 / \alpha$ according to eq 4 furnishes a family of straight lines with $f$ as a parameter. The values of these straight lines in Figure 1 were obtained assuming $\Delta h_{\mathrm{f}}=2.8 \times 10^{9}$ $\mathrm{erg} / \mathrm{cm}^{3}, \quad T_{\infty}=141^{\circ} \mathrm{C}, \sigma=80 \mathrm{erg} / \mathrm{cm}^{2}, \rho_{\mathrm{c}}=1$ and values of $f$ as shown. The numerous experi-



Figure 1. Melting point, $T(l)$, against inverse of crystallinity, $1 / \alpha: \nabla, \mathrm{PE}$ single crystals; ${ }^{19}$ meltcrystallized material, $\bigcirc$, with high molecular weight, - low molecular weight. The crosses are the data from Tanaka ${ }^{18}$ quoted by Knox. ${ }^{1}$ The solid straight lines are calculated from eq 4 , using values of $f$ as shown.

mental data obtained by Illers and Hendus ${ }^{9}$ and Peterlin and Meinel ${ }^{10}$ for melt- and solutioncrystallized and drawn PE samples with wide varying branching concentration and molecular weight values and known crystal thickness justify the chosen value of $\sigma=80 \mathrm{erg} / \mathrm{cm}^{2}$.

\section{EXPERIMENTAL}

The materials investigated were a series of commercial samples (see Table I) with greatly varying molecular weight and branching content values. The samples were crystallized from the melt by rapid cooling in air at room temperature.

The density, $\rho$, of the samples was measured in acetone by the flotation method ${ }^{11}$ at $20^{\circ} \mathrm{C}$ with an uncertainty of $0.5 \%$. The crystallinity, $\alpha$, was estimated from density values, assuming the additivity of densities in the crystalline and amorphous phases according to Kilian: ${ }^{12}$

$$
\alpha=\frac{\rho_{\mathrm{c}}}{\rho}\left(\frac{\rho-\rho_{\mathrm{a}}}{\rho_{\mathrm{c}}-\rho_{\mathrm{a}}}\right)
$$

A value of $\rho_{\mathrm{a}}=0.86 \mathrm{~g} / \mathrm{cm}^{3}$ was taken for the density of the amorphous phase. ${ }^{13}$ The crystal (unit cell) density $\rho_{c}$, was derived from the volume of the unit cell. For this purpose the 
Table I.

\begin{tabular}{|c|c|c|c|c|c|c|c|c|}
\hline & $\begin{array}{l}\text { Average } \\
\text { molecular } \\
\text { Weight }\end{array}$ & $\begin{array}{l}\text { Density } \\
\rho, \mathrm{g} / \mathrm{cm}^{3}\end{array}$ & $\begin{array}{c}\text { Unit cell } \\
\text { density } \\
\rho_{\mathrm{c}}, \mathrm{g} / \mathrm{cm}^{3}\end{array}$ & $\begin{array}{c}\text { Melting } \\
\text { point } \\
T(l),{ }^{\circ} \mathrm{C}\end{array}$ & $\begin{array}{c}\text { Degree of } \\
\text { branching, } \\
\mathrm{CH}_{3} / 100 \mathrm{C}\end{array}$ & $\begin{array}{c}\text { Crystal- } \\
\text { linity } \\
\alpha_{\rho}\end{array}$ & $\begin{array}{c}\text { Crystal } \\
\text { thickness } \\
l, \AA\end{array}$ & $f$ \\
\hline Marlex 6015 & $15.0 \times 10^{4}$ & 0.980 & 0.988 & 131 & 0.05 & 0.94 & 239 & 12 \\
\hline Hostalen GC & 5.0 & 0.969 & 0.994 & 129 & 0.10 & 0.83 & 198 & 32 \\
\hline Hostalen GF & 12.0 & 0.968 & 0.989 & 128 & 0.64 & 0.85 & 184 & 27 \\
\hline Hoechst PA-190 & 0.9 & 0.954 & 0.988 & 124 & 0.84 & 0.76 & 141 & 35 \\
\hline Alkathene HD & 24.5 & 0.952 & 0.986 & 121 & 0.91 & 0.76 & 120 & 30 \\
\hline Epolene C-11 & 1.0 & 0.947 & 0.981 & 119 & 1.08 & 0.74 & 110 & 30 \\
\hline Lupolen KR 1051 & 5.1 & 0.943 & 0.984 & 120 & 1.30 & 0.70 & 114 & 38 \\
\hline BASF a Wachs & 1.0 & 0.933 & 0.973 & 111 & 1.84 & 0.67 & 81 & 31 \\
\hline Lupolen KR 1032 & 5.3 & 0.931 & 0.986 & 115 & 2.50 & 0.60 & 92 & 49 \\
\hline Epolene C-13 & 1.0 & 0.919 & 0.977 & 104 & 2.80 & 0.54 & 65 & 44 \\
\hline Lupolen $1810 \mathrm{H}$ & 5.4 & 0.928 & 0.982 & 106 & 3.00 & 0.59 & 69 & 37 \\
\hline Hoechst PA-130 & $3.0 \times 10^{3}$ & 0.957 & 0.976 & 115 & 1.58 & 0.85 & 93 & 13 \\
\hline Epolene $\mathrm{N}-10$ & 2.5 & 0.925 & 0.973 & 105 & 2.25 & 0.61 & 68 & 34 \\
\hline Epolene N-12 & 1.5 & 0.941 & 0.972 & 108 & 2.27 & 0.75 & 74 & 19 \\
\hline BASF a Wachs & 5.6 & 0.936 & 0.977 & 106 & 2.32 & 0.68 & 69 & 26 \\
\hline Epolene $\mathrm{N}-11$ & 1.5 & 0.934 & 0.974 & 104 & 2.48 & 0.68 & 66 & 24 \\
\hline Hoechst PA-560 & 6.0 & 0.914 & 0.973 & 101 & 4.10 & 0.51 & 61 & 46 \\
\hline Epolene C-10 & 7.0 & 0.919 & 0.964 & 99 & 4.19 & 0.60 & 58 & 30 \\
\hline Epolene C-101 & 3.2 & 0.936 & 0.973 & 104 & 4.42 & 0.70 & 66 & 22 \\
\hline Hoechst PA-520 & 2.0 & 0.917 & 0.972 & 102 & 4.90 & 0.54 & 62 & 42 \\
\hline Epolene C-12 & 3.7 & 0.906 & 0.957 & 83 & 6.94 & 0.50 & 51 & 33 \\
\hline
\end{tabular}

$a$ and $b$ unit cell dimensions were calculated from the location of the 110 and 200 reflections measured by Mr. J.C. Gonzalez Ortega ${ }^{14}$ in this laboratory from X-Ray diffractometric scans. The cell parameter, $c$, along the chain direction was assumed to be equal to $2.547 \AA .^{15}$ The standard errors in the cell dimensions correspond to a standard deviation of $0.001 \mathrm{~g} / \mathrm{cm}^{3}$ per unit cell density.

The melting points were determined on a temperature gradient bar. Since this technique involves a fast heating rate it minimizes recrystallization effects and justifies the use of eq 3 . The branching content of the samples was investigated by means of IR analysis. ${ }^{16}$ The band of methyl symmetrical deformation at $1378 \mathrm{~cm}^{-1}$ was selected for this purpose. The samples were calibrated with a series of standard samples with known amounts of methyl concentration. ${ }^{17}$

\section{RESULTS AND DISCUSSION}

The measured macroscopic and crystal densities, melting point and branching content, and the crystallinity calculated from density data (eq 5 ), the crystal thickness, $l$, from eq 3 taking into account the $\rho_{\mathrm{c}}$ measured values, and the number of noncrystalline backbone units per fold, $f$, from Figure 1 are collected in Table $I$ into two major groups according to the number average molecular weight. The $f$ values offered are a function of crystallinity and consequently should be taken as a tentative estimate since they may obviously vary according to the physical definition of crystallinity used. The data of Tanaka ${ }^{18}$ quoted by $\operatorname{Knox}^{1}$ are shown in Figure 1 as crosses. These values correspond to $\mathrm{PE}$ samples slowly cooled from the melt at room temperature with a $M_{n}=1.2-5 \times 10^{4}$. The crystallinity of these samples was calculated from specific-volume dilatometric measurements by using an expression equivalent to eq $5 .^{18}$ "Single crystal" data ${ }^{19}$ of various linear PE fractions crystallized for various times at different temperatures are also plotted for comparison. In this case, $\alpha$ was calculated from the density data given, ${ }^{19}$ assuming $\rho_{\mathrm{c}}$ equal to unity in eq 5 . The crystal thickness was derived from $l=L \alpha, L$ being the X-Ray long 
period, and $T(l)$ was calculated from eq 3 .

By simple inspection of Figure 1 one sees that the collected data are scattered over the entire $(T(l), 1 / \alpha)$ plot. Contrary to Knox's contention, $(f=40)$, the present results show that $f$ can vary between 5 and 60 depending on crystallization conditions, branching concentration, and molecular weight. In addition, the present model does provide low crystallinity values which are quantitatively consistent with a large contribution of noncrystalline backbone units per fold. The lowering of $T(l)$, and consequently of $l$, for the melt-crystallized samples as $\alpha$ decreases is apparently connected with the increase in branching concentration (see Table I and ref 18). Thus, the increase of the number of chain defects (branching) not only provokes a clear decrease of crystal thickness but also can be correlated, in accordance with previous results of Kawai, ${ }^{20}$ with an increase in the number of noncrystalline backbone units per fold. The latter trend in $f$ as a function of branching concentration for the melt-crystallized samples can be seen in Table I, with higher molecular weights roughly yielding larger $f$ values. For low branching concentrations (linear PE) one expects an additional dependence of $f$ on crystallization conditions.

The data plotted for single crystals in Figure 1 clearly show lower $f$ values than those for the majority of melt-crystallized samples. It is interesting to note, however, that a few meltcrystallized linear PE samples for example, $\mathrm{N}-12$, PA-130, Marlex 6015, and Tanaka's with $T(l)=$ $136^{\circ} \mathrm{C}$ and $0.2 \mathrm{CH}_{3} / 100 \mathrm{C}$, do exhibit $f$ values which are comparable with those obtained for single crystals. In one instance at least, (fraction $P_{n}=1010$ crystallized at $77.2^{\circ} \mathrm{C}$ during $44 \mathrm{hr}^{19} f$, fits with a value of 5 methylenes per fold, thus favouring the idea of a surface layer with sharp folds. According to this result a 5-\% crystallinity deficiency would be at least compatible with regular sharp folds. The remaining single crystal data show $f$ values ranging between 7 and 20. It is worthwile noting that these values represent the highest possible estimate for $f$, since they were derived assuming $\rho_{\mathrm{c}}=1$ in eq 5. $\rho_{\mathrm{c}}$ values smaller than unity for linear PE single crystals, as those for example measured by other authors, ${ }^{15}$ would undoubtedly result in a shift of the data of Figure 1 towards lower $1 / \alpha$ values, i.e., towards lower $f$ values. If one would admit, however, according to the present data loose loops of, say, 15-20 carbon atoms and adjacent reentry one would certainly be faced with lack of space for the amorphous conformation, which needs an appreciably larger cross section for every chain as compared with the crystalline lattice. ${ }^{7}$ Hence, if, as suggested previously, ${ }^{6,7,21}$ a regularly folded structure with variable amounts of additional disorder is assumed one would solve the problem of space and entropy. According to this view, $f$ in the present approach cannot be strictly visualized as the number of noncrystalline methylene groups per fold but it should be rather interpreted as an average value. A hypothetical case with $f=10$, for example, could be explained either as one loose loop of 50 backbone units every 10 sharp regular folds or as two loops of 25 units every 10 regular folds or as any other combination which would be compatible with the presence of adjacent sharp and loose loops.

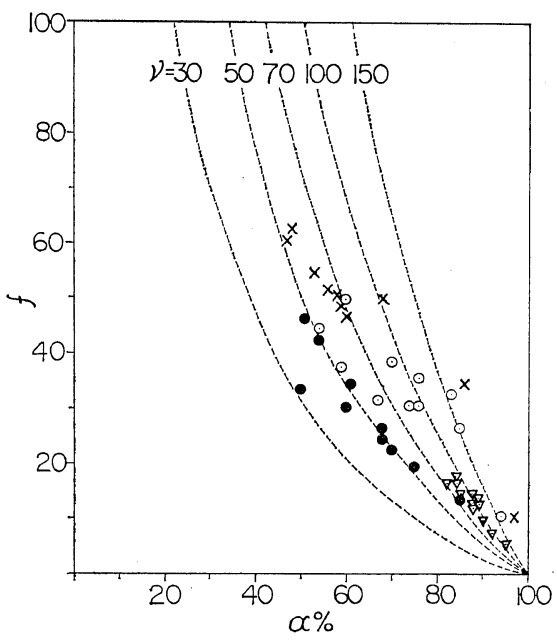

Figure 2. Number of noncrystalline backbone units per fold, $f, v s$. crystallinity, $\alpha$, for PE: $\nabla$, single crystals; ${ }^{19}$ melt-crystallized material, $\bigcirc$, with high molecular weight, $\bullet$, with low molecular weight, $\times$, data of Tanaka. ${ }^{18}$ The broken curves were calculated from eq 6 using $\nu$ values as shown.

Figure 2 shows the clear dependence between the average number of backbone units per fold and crystallinity. The trend is here consistent with the fact that $f$ must tend towards infinity 
when $\alpha$ approaches zero. This can be straightforwardly seen if one writes eq 1 as:

$$
f=\frac{\nu(1-\alpha)}{\alpha}
$$

This expression explicitly shows the expected dependence of $f$ as a function of $\alpha$, with $\nu$ as parameter, according to the suggested model. By comparison of the experimental data with the broken curves of eq 6, one sees in Figure 2 a clear separation of $f$ values depending on the crystal thickness selected. An apparent segregation of data according to molecular weight can be also observed in this plot. Thus, for a given crystallization mode, it seems that PE samples with lower molecular weight tend to exhibit smaller $l$ values than those with higher molecular weight.

Since the present analysis of melting point and crystallinity is based on the assumption that the amorphous content is localized at the fold surface it is interesting to verify whether this approach is consistent with typical annealing data which unambigously favor such an assumption. ${ }^{22}$ In Figure 3 the data derived from the work of Fischer and $\mathrm{Schmidt}^{22}$ for annealed single crystals are plotted for comparison with the values obtained from the present model (solid lines). For single crystals before annealing, one obtains $f=$

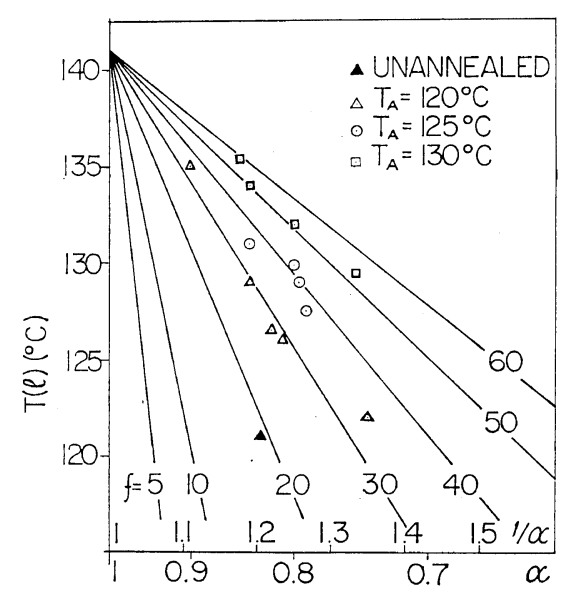

Figure 3. Melting point, $T(l)$, vs. inverse of crystallinity, $1 / \alpha$, for PE single crystals annealed at constant temperature for various lengths of time. The data in this plot are derived from the results of Fischer and Schmidt.22
20, a value which is close to the other single crystal data shown in Figure 1. The successive annealing of the crystals at 120,125 , and $130^{\circ} \mathrm{C}$ provokes an increase in $f$ to values of 30,40 , and 50, respectively. Thus, the derived melting points of annealed crystals from ref 22 against $1 / \alpha$ yield a series of straight lines which resemble the well known plot relating $\rho$ with the inverse of the long period, $L:^{22}$

$$
\rho=\rho_{\mathrm{c}}-A / L
$$

This is most probably due simply to the fact that $\rho$ is proportional to $\alpha$ and $1 / L$ is inversely proportional to $T(l)$. Hence both representations are equivalent, the $(T(l), 1 / \alpha)$ plot being the inverse of the $(\rho, 1 / L)$ relationship. As a consequence, the constant $A$ and the slope of the $(T(l), 1 / \alpha)$ plot, which is also dependent upon annealing temperature, seem to have a similar physical significance, i.e., the amount of crystallinity defect between crystals, though the type of information yielded in each case is slightly different. Nevertheless, the conclusions drawn from the present analysis are essentially the same as those derived from density and X-ray long spacing $\mathrm{data}^{22}$ and consequently justify the approach used.

To sum up, the model suggested in the present work provides an estimate of the average number of backbone units per fold which is in fairly close agreement with experimental data obtained by other methods. The results obtained by Kawai, et al. ${ }^{23}$ from fumic nitric acid experiments on single crystals and melt-crystallized material quantitatively confirm our $f$ derived values on similar samples.

We wish to thank Mr. J. C. Gonzalez Ortega for contributing his X-ray diffraction data, as mentioned in the next.

\section{REFERENCES}

1. J. R. Knox, J. Polym. Sci., Part C, 18, 69 (1967).

2. G. Adam, Kolloid. Z., 180, 11 (1962).

3. J. B. Jackson, P. J. Flory, and R. Chiang, Trans. Faraday Soc., 49, 1906 (1963).

4. J. Loboda-Ćaćković, H. Ćaćković, and R. Hosemann, J. Polym. Sci., in press.

5. R. Hosemann, W. Wilke, and F. J. Baltá Calleja, Acta Cryst., 21, 118 (1966).

6. A. Keller, Kolloid. Z., 231, 386 (1969). 
Study of the Interlamellar Folded Structure of Polyethylene

7. A. Peterlin, J. Macromol. Sci. Phys., B 3, 11, 19 (1969).

8. T. Kawai, Kolloid. Z., 201, 15 (1965).

9. K. H. Illers and H. Hendus, Makromol. Chem., 113, 1 (1968).

10. G. Meinel, N. Morosoff, and A. Peterlin, $J$. Polym. Sci., Part A-2, 8, 1723 (1970).

11. F. J. Baltá Calleja and A. Schönfeld, Faserforsch. u. Textiltech., 18, 1970 (1967).

12. H. G. Kilian, Kolloid. Z., 176, 49 (1961).

13. H. Hendus and G. Schnell, Kunstoffe, 51, 69 (1961).

14. J. C. Gonzalez Ortega, (unpublished results).

15. G. J. Davies, R. K. Eby, and G. M. Martin, J. Appl. Phys., 39, 4973 (1968).

16. D. R. Rueda, A. Hidalgo, and F. J. Baltá
Calleja, Paper presented at the 16th Meeting of the "Real Soc. Esp. Fís. Quím.", Oviedo, Spain, Sept., 1973.

17. F. J. Baltá Calleja and A. Hidalgo, Kolloid. Z., 229, 21 (1969).

18. K. Tanaka, Bull. Chem. Soc. Japan, 33, 1060 (1960).

19. A. Nakajima and S. Hayashi, Kolloid. Z., 225, 116 (1968).

20. T. Kawai, K. Ujihara, and H. Maeda, Makromol. Chem., 132, 87 (1970).

21. T. Kawai, ibid., 90, 288 (1966).

22. E. W. Fischer and G. F. Schmidt, Angew. Chem., 74, 551 (1962).

23. T. Kawai, T. Goto, and H. Maeda, Kolloid. $Z$., 223, 117 (1968). 